\title{
Quantitative analysis of task selection for Brain-Computer Interfaces
}

\author{
Alberto Llera*, ${ }^{*}$, Vicenç Gómez ${ }^{*}, 1,2$, and Hilbert J. Kappen ${ }^{1}$ \\ ${ }^{1}$ Donders Institute for Brain, Cognition and Behaviour \\ Radboud University Nijmegen, the Netherlands \\ a.lleraedonders.ru.nl, b.kappenescience.ru.nl \\ ${ }^{2}$ Department of Information and Communication Technologies \\ Universitat Pompeu Fabra. Barcelona, Spain \\ vicen.gomez@upf.edu
}

\begin{abstract}
Objective: To assess quantitatively the impact of task selection in the performance of Brain Computer Interfaces (BCI). Approach: We consider the task-pairs derived from multiclass BCI imagery movement tasks in three different datasets. We analyze for the first time the benefits of task selection on a large-scale basis (109 users) and evaluate the possibility of transferring task-pair information across days for a given subject. Main results: Selecting the subject-dependent optimal task-pair among three different imagery movement tasks results in approximately $20 \%$ potential increase in the number of users that can be expected to control a binary BCI. The improvement is observed with respect to the best task-pair fixed across subjects. The best task-pair selected for each subject individually during a first day of recordings is generally a good task-pair in subsequent days. In general, task learning from the user side has a positive influence in the generalization of the optimal task-pair, but special attention should be given to inexperienced subjects. Significance: These results add significant evidence to existing literature that advocates task selection as a necessary step towards usable BCIs. This contribution motivates further research focused on deriving adaptive methods for task selection on larger sets of mental tasks in practical online scenarios.
\end{abstract}

Keywords: Brain Computer Interface, Task selection, Subject learning.

\footnotetext{
${ }^{*}$ Authors contributed equally.
} 


\section{Introduction}

Brain Computer Interfaces (BCI) [41] aim to provide humans with control over devices or computer applications only through their measured brain activity by e.g. electroencephalogram (EEG) [20]. The automatic on line discrimination of mental tasks could be used to provide the user with an extra channel of communication/action that could for example be used to improve the quality of life of severely disabled people [46].

A common paradigm in BCI is induced modulations (IM), which rely on voluntarily induced user brain activity modulations, for instance, imaginary movement [33], mental counting, writing or rotating a $3 \mathrm{D}$ object $[24,13]$ or the covert attention paradigm [40].

Although BCI research has so far not delivered a reliable technology for widespread applications, the progress in EEG-based BCI technology in the last decade has been undoubtedly significant. For example, in comparison with a study from year 2003 in which $41 \%$ of the analyzed subjects showed satisfactory BCI control [19], a more recent study from year 2012 shows that BCI control was reached by $60 \%$ of the subjects [21] ${ }^{1}$.

Clearly several factors have contributed to such performance improvement: development of better feature extraction algorithms and optimization techniques, advances in hardware, on line BCI adaptation, etc. Among all the methodological advances achieved, however, a relatively unexplored question is whether task selection constitutes a key factor for such improvement.

Task selection is being progressively introduced in some BCI systems. In [14] an exploratory step was used to select useful sets of subject dependent imagery movement tasks for a multi-class problem. In [7] it was shown that untrained subjects can achieve effective binary performance with a short training period by using advanced machine learning techniques. In that case, the optimization procedure included the detection of the best task-pair between right hand, left hand or feet imagery.

Recent off line experiments [13] have shown the benefits of task selection for a small set of BCI users using a large set of non-motor tasks. The use of distinct mental strategies combined with motor imagery tasks may lead to increased performance, as shown in [16] using nine users and seven non-motor mental tasks. In [21] the imagery movement task-pair was optimized between three different motor imagery movement tasks.

In this work, we explore for the first time the benefits of task selection on a large number of users and whether the optimal task-pair remains optimal across multiple days, or needs to be

\footnotetext{
$1_{\mathrm{A}}$ threshold of $70 \%$ correct classification during on line sessions is considered necessary for the user to feel a minimum of control over a binary BCI device [27, 42].
} 
re-estimated on every single session. Based on our results, we claim that the design of a BCI has to consider task selection as a necessary step.

In the next section we present the datasets and the methods considered in this study. We then present our results in section 3. We conclude the paper in section 4 with discussion and future work.

\section{Methods}

We use three different datasets composed of multi-class imagery movement tasks.

\subsection{Datasets}

Dataset A: (Physiobank [18, 36]). This dataset contains data from 109 subjects performing various combinations of real and imagined movements in one day of recordings. In this work we consider only the data related to left hand, right hand and both feet imagery movement. Each subject performed 22 trials of each class and the EEG data were recorded from 64 electrodes. Due to the computational load required by the extended analysis presented in this work, we focus only on 20 EEG electrodes over the sensorimotor cortex. The large number of users makes this dataset convenient to evaluate the impact of task selection on a large scale basis.

Dataset B: (BCI competition IV, 2a) ${ }^{2}$ : This dataset [38] provides data from 9 subjects performing 4 different imagery movement tasks (right hand $(\mathrm{RH})$, left hand $(\mathrm{LH})$, both feet $(\mathrm{F})$, tongue (T)) during 2 different days of recordings. Each day they performed 72 trials of each task and the EEG data were recorded from 20 electrodes. The fact that it contains two different days of recordings allows to analyze the influence of time in relation with task selection.

Dataset C: (BSI RIKEN) ${ }^{3}$ : This dataset provides data from several subjects performing binary or multi-class imagery movement tasks. We only consider the 2 subjects performing multiclass problems (LH, RH, F).

\footnotetext{
2 BCI Competition 2008 - Graz data set A. Available at http://www .bbci.de/competition/iv/desc_2a.pdf.

3 Available at http://www.bsp.brain.riken.jp/ qibin/homepage/Datasets.html.
} 
Subject 1 was recorded on 2 different days and Subject 2 in seven different days. Each day they performed $\approx 65$ trials (3-4 seconds) of each task and the EEG data were recorded using 5 or 6 electrodes. In this work we always consider the same 5 EEG electrodes, C3, Cp3, C4, Cp4 and Cz.

For subject 2 there is a previous additional recording day consisting of three long sessions $(\approx 268$ trials per session) distributed along a different day. We denote these data as subject $2 \mathrm{~b}$. There are long periods between the sessions and each session contains considerably more trials than the other seven days of recordings. Hence, subject $2 b$ is considered as an independent dataset and each session will be treated as an independent day.

We focus on binary classification problems and consider all possible pairs of different binary problems for each dataset and subject. This results in 3,6 and 3 possible task-pairs for each subject in datasets $\mathrm{A}, \mathrm{B}$ and $\mathrm{C}$, respectively.

\subsection{Preprocessing, feature extraction and classification}

The raw data were detrended and bandpass-filtered in the frequency bands 8-30 Hz. The features for classification were extracted using the Tangent Space Mapping (TSM) [3, 29]. This is a recently proposed method that has comparable or better performance than more typical algorithms such as Common Spatial Patterns [34, 3, 2].

Noting that covariance matrices belong to the Riemannian manifold of symmetric positivedefinite matrices $(\mathcal{M})$ [31], TSM is a non-linear projection of the spatial covariance matrices of the data into the tangent space [12] of $\mathcal{M}$ at the Riemannian (or geometric) mean [22] of the spatial covariance matrices of the training data. The Riemannian mean $\left(\mathcal{C}_{\mathcal{R}}\right)$ of a set of covariance matrices $\left\{C_{1}, \ldots, C_{n}\right\} \in \mathcal{M}$ is defined as

$$
\mathcal{C}_{\mathcal{R}}=\operatorname{argmin}_{C \in \mathcal{M}} \sum_{k=1}^{n} d_{\mathcal{R}}\left(C_{k}, C\right)^{2}
$$

where $d_{\mathcal{R}}:(\mathcal{M} \times \mathcal{M}) \rightarrow \mathcal{R}^{\geq 0}$ denotes the Riemannian distance induced by the Riemann geometry on $\mathcal{M}$ and it can be computed as a generalized eigenvalue problem [30]. More precisely, for any two $C_{k_{1}}, C_{k_{2}} \in \mathcal{M}$

$$
d_{\mathcal{R}}\left(C_{k_{1}}, C_{k_{2}}\right)=\left[\sum_{i=1}^{m} \log ^{2} \lambda_{i}\right]^{1 / 2}
$$


where $\lambda_{i}, i \in\{1, \ldots, m\}$, are the eigenvalues of $C_{k_{1}}^{-1} C_{k_{2}}$.

The geometric mean exists within the considered manifold $(\mathcal{M})$, and it is unique [23]. Although there is no closed-form solution for its computation, it can be computed efficiently using iterative algorithms. In this work we consider the algorithm presented by [15].

Given a set of covariance matrices and denoting their geometric mean as $\mathcal{C}_{\mathcal{R}}$, the tangent space mapping at $\mathcal{C}_{\mathcal{R}}$ of a given covariance matrix $C_{k}$ is given by

$$
\operatorname{TSM}_{\mathcal{C}_{\mathcal{R}}}\left(C_{k}\right)=\log \left(\mathcal{C}_{\mathcal{R}}{ }^{\frac{-1}{2}} C_{k} \mathcal{C}_{\mathcal{R}}{ }^{\frac{-1}{2}}\right)
$$

where the log is the logarithm of a matrix derived from its diagonalization [4].

$\operatorname{TSM}_{\mathcal{C}_{\mathcal{R}}}\left(C_{k}\right)$ is a symmetric matrix that, in vectorized form (after eliminating redundant elements due to symmetry) can be used as features for classification in BCI problems [3]. Since one unique $\mathcal{C}_{\mathcal{R}}$ is computed using all of the covariances matrices in the training set, TSM is an

unsupervised feature extractor for covariance classification ${ }^{4}$. We used regularized Linear Discriminant Analysis [8] as classifier.

Note that we excluded an exhaustive optimization at the level of individual frequency bands. We expect such optimizations to provide a general improvement. The reason to omit them was to focus on the task selection effect, which is the main interest of this paper.

\subsection{Assessment of BCI control performance}

We measure BCI control performance in terms of classification rate, defined as the number of correctly classified trials divided by the total number of trials. The generalization performance of a task-pair is estimated using cross validation on hold-out data (CV) [6]. We use leave-one-out $\mathrm{CV}$ and denote $C V$ rate as one minus the $\mathrm{CV}$ error.

For each subject, we define $C V_{d}\left(t_{i}\right)$ as the $\mathrm{CV}$ rate considering a classifier trained on the $i$-th task-pair in day $d$. Further, we will use $t_{d}^{*}$ to denote the optimal task-pair for a specific day $d$, i.e. the task-pair that gives the highest $\mathrm{CV}$ rate.

Finally, we will use Wilcoxon statistical tests [11] to assess statistical significance between different performances. We consider significance level of $\alpha=0.05$.

\footnotetext{
${ }^{4}$ We used the Covariance Toolbox from https://github.com/alexandrebarachant/covariancetoolbox.
} 


\section{Results}

In this section we first analyze the potential improvement that can be expected at the population level from selecting the subject-dependent optimal task-pair. Then we consider the optimality of a task-pair across different days of recordings and study how task learning from the subject side can influence the task selection step.

\subsection{Task optimization significantly improves BCI control}

We consider imagery movement based BCI using the dataset A, which consists of three tasks and a large number of subjects. To quantify the potential improvement that can be achieved selecting the optimal task-pair for each BCI user, we compare the probability distributions of the CV rates for each task-pair independently against the CV rate that is obtained with the best task-pair for each user.

Figure 1 shows histograms (left) and the corresponding cumulative distribution functions (right). These probability distributions show the large variance associated to the subject heterogeneity. Notice that left versus right hand imagery movement task ( $\mathrm{LH}$ vs $\mathrm{RH}$, top-left panel) is the task-pair that globally results in the worst performance with approximately $30 \%$ of the users performing at around random guess. The task-pairs involving one of the hands versus foot imagery have very similar probability distributions and are more skewed toward higher CV rates.

The mean task-pair CV rates across subjects were $0.64,0.81$, and 0.80 for $\mathrm{LH}$ vs RH, LH vs $\mathrm{F}$ and $\mathrm{RH}$ vs F, respectively. In contrast, selecting subject dependent optimal task-pairs resulted in a mean value of 0.88 , a significant improvement in performance with respect to any of the fixed task-pair choices. If we consider the proportion of users providing task-pairs with $\mathrm{CV}$ rates within the range $80 \%-90 \%$, a remarkable improvement of approximately $20 \%$ is observed.

These results clearly show that merely user-specific task selection results in a dramatic increase on the number of BCI users potentially able to provide highly discriminative brain features. Although this is a remarkable improvement, its generalization to an on line feedback setting has to be done with caution. In the presence of strong nonstationarities, a proportion of users may fail to achieve effective control in testing/feedback sessions despite being able to provide discriminative features during training $[25,9,21]$. 

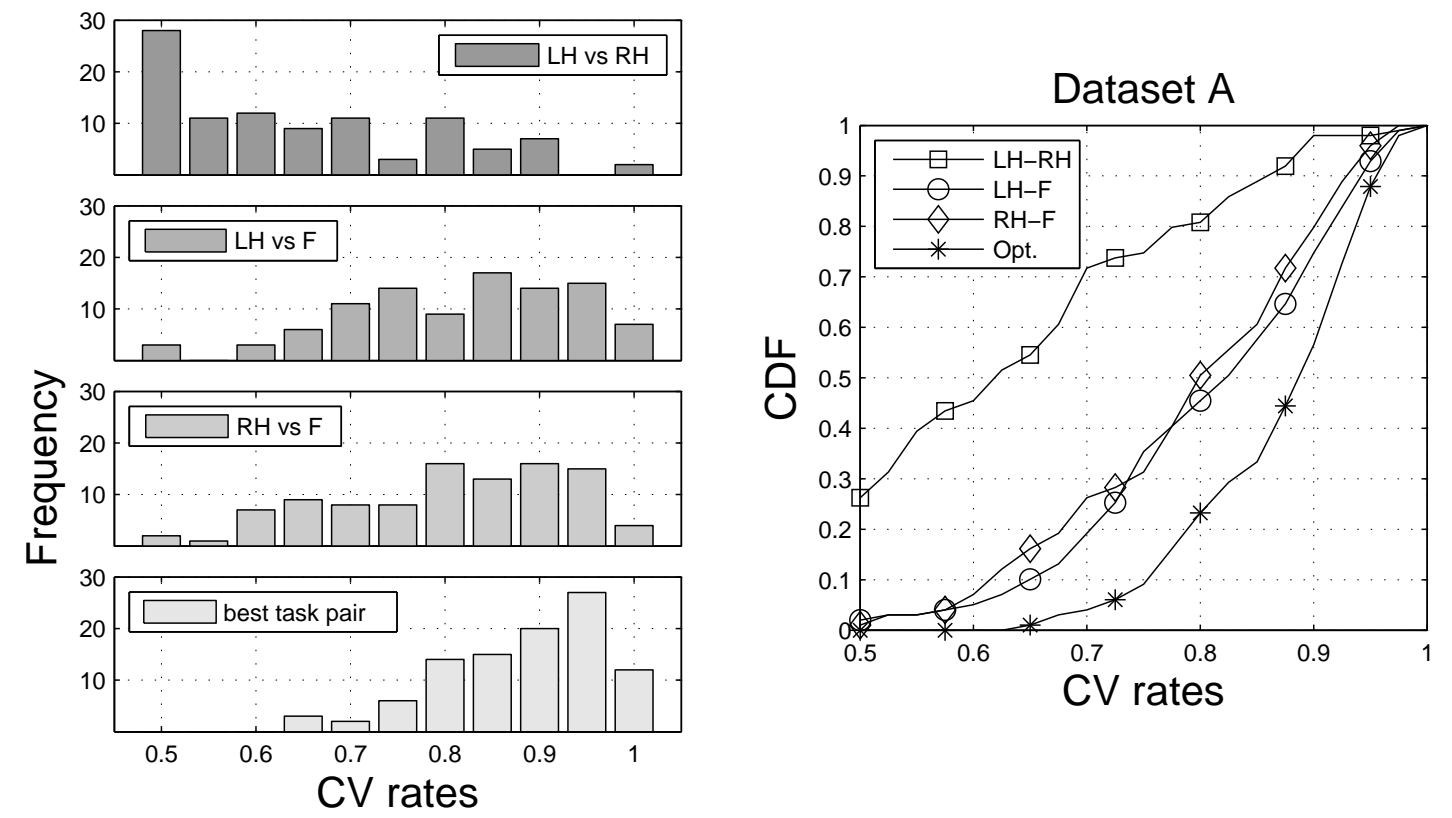

Figure 1: Effect of task selection in binary imaginary movement BCI control: data corresponding to 109 subjects and three different task-pairs (Dataset A). Left: histograms of the Cross-Validation rates for the three different task-pairs and the one obtained using the subject dependent optimal task-pair (bin size $=0.025$ ). Right: estimated cumulative distribution function of the Cross-Validation rates for each task-pair together with the results selecting the optimal task-pairs (curve with asterisks). Significant improvements are obtained using task selection (p-value $<$ $10^{-10}$ in all three tests between each task-pair against the best task-pair across subjects).

\subsection{Generalization of the optimal task-pair across days}

So far we have seen that selecting a subject dependent optimal task-pair may result in a dramatic improvement in BCI control performance. However, partly due to the non-stationary character of the EEG signal $[37,25]$, there is no guarantee that the optimal task-pair in one day remains optimal also for subsequent sessions or days. If that is not the case, how big is the performance loss if we select the suboptimal task-pair? Is significantly better to choose the task-pair with best performance in the past, or does not make any difference when compared, for instance, with random task-pair selection?

To answer these questions, we first characterize the optimal task-pair in different days. Figure 2 shows histograms corresponding to the two days of dataset B. They show large variability 

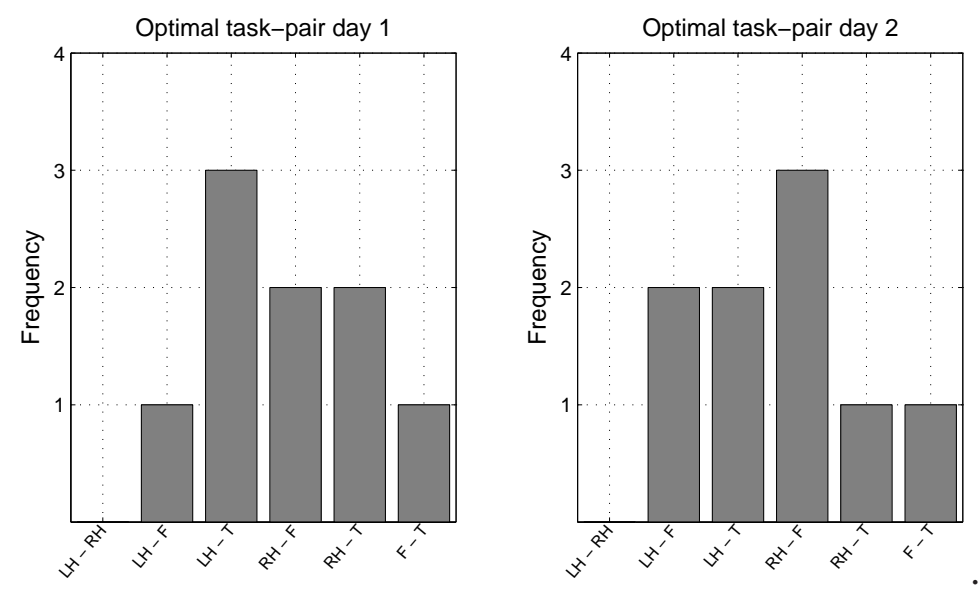

Figure 2: Dataset B: frequency (number of subjects) of the optimal task-pair for each of the 6 possible task-pairs on each day of recordings.

across subjects. Interestingly, the task left/right hand (LH-RH) is never optimal. This result is in agreement with the conclusion previously reported for dataset A and previous literature [32].

We also observe that tongue imagery movement task (T) appears frequently in the optimal task-pair, indicating a high discriminative power. This is interesting because $\mathrm{T}$ is not usually considered in binary motor imagery BCI systems.

We now consider both $\mathrm{B}$ and $\mathrm{C}$ datasets (with eleven subjects in total) and compare, for each subject individually, the performances of each task-pair $i$ between the first day of recordings $C V_{1}\left(t_{i}\right)$ and a posterior day of recordings $C V_{k}\left(t_{i}\right), k>1$. Figure 3 shows this comparison with a different panel for each case. Each dot indicates a task-pair. In total, the optimal task-pair on the first day remains optimal on a subsequent day/session in nine out of the eighteen cases. These are marked with a cross in the optimal task-pair.

Four out of ten subjects participating in just two days of recordings present a constant optimal task-pair with respect to the first day. Considering the subject participating in several days (C$\mathrm{S} 2$ ), the optimal task-pair changes over days (third row, column 5 and bottom row of Figure 3) and also within the sessions in a single day (C-S2b, third row, columns 1 and 2).

Globally, we observe that users tend to learn to better execute the tasks $[10,42]$. This effect is likely to be present when most of the dots fall above the diagonal. We quantify such learning effect by taking the average of the differences in performance of each task. This value is shown in the inset as learn (a positive value indicates learning). We observe that there is a general improvement if we globally compare all differences in performance $\left(\mathrm{p}\right.$-value $\left.<10^{-4}\right)$. It is interesting to 


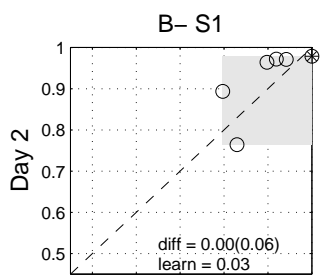

Day 1

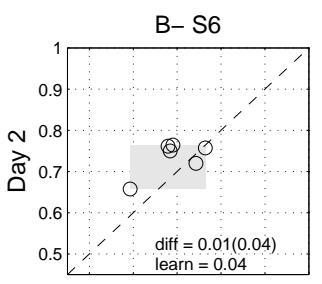

Day 1

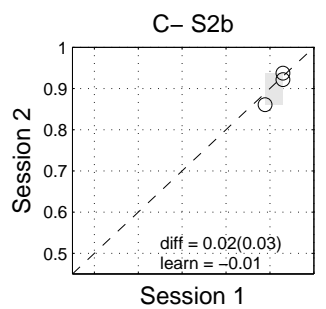

C- S2

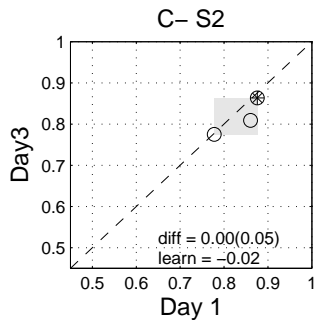

B- S2

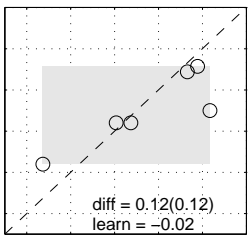

B- S7

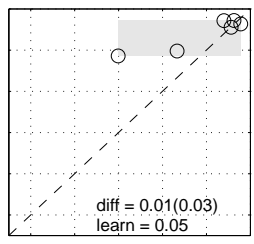

C- S2b

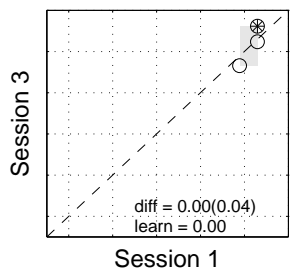

$\mathrm{C}-\mathrm{S} 2$

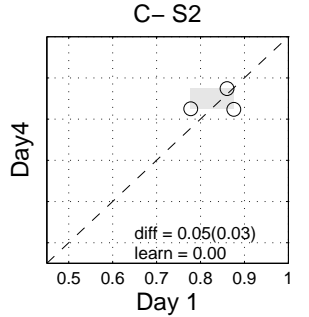

B-S3

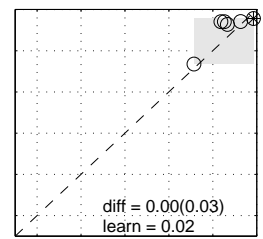

B- S8

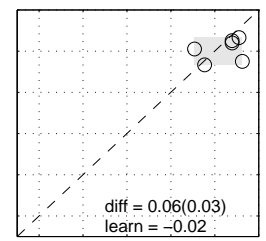

diff $=0.06(0.03$
learn $=-0.02$

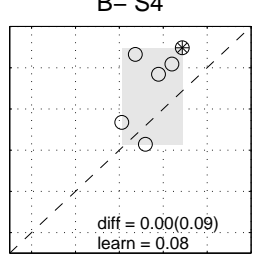

B- S9

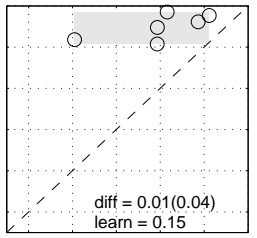

learn $=0.15$

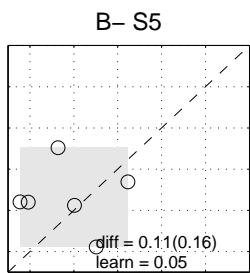

C-S1

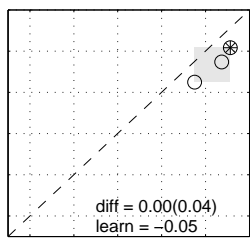

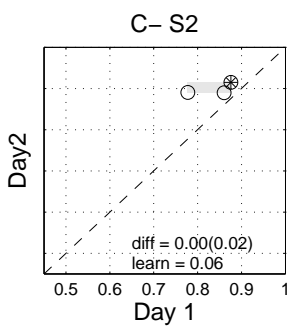
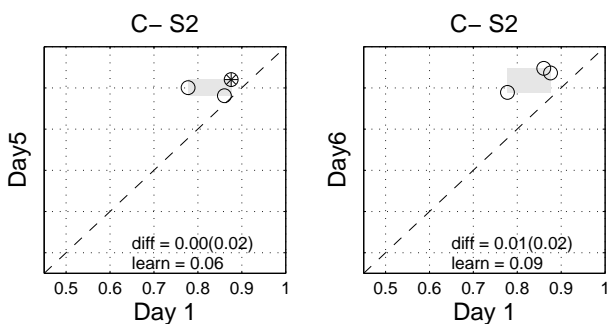

Figure 3: Generalization of optimal task-pairs. Each panel corresponds to one subject and shows a comparison between the performance on each task-pair $i$ between the first day of recordings $C V_{1}\left(t_{i}\right)$ (x-axis) and a posterior day $k>1, C V_{k}\left(t_{i}\right)$ (y-axis). Subject number is preceded by dataset name. Inset diff $:=C V_{k}\left(t_{k}^{*}\right)-C V_{k}\left(t_{1}^{*}\right)$ denotes the loss incurred when the optimal task-pair of the first day $t_{1}^{*}$ is selected instead of the unknown optimal one $t_{k}^{*}$. The value between brackets indicates the loss incurred if the task-pair is selected randomly and is computed as $\frac{1}{6} \sum_{i=1}^{6} C V_{k}\left(t_{k}^{*}\right)-C V_{k}\left(t_{i}\right)$. Inset learn $:=\frac{1}{6} \sum_{i=1}^{6} C V_{k}\left(t_{i}\right)-C V_{1}\left(t_{i}\right)$ represents the learning from the user to better execute the task from day 1 to day $k$. Grey rectangular areas on each subplot denote performance ranges.

notice the ability of some subjects to improve on task-pairs that were clearly suboptimal during the first day, e.g. subjects B-S4, B-S5, B-S7, B-S9. Further, in most cases the variance across task-pairs is also reduced (compare the sides of the grey areas). These facts suggest that learning 
from the user side could have a strong effect in the selection of a task-pair using data from a previous recording day.

How bad is the performance on a day $k$ if the optimal task-pair on the first day $t_{1}^{*}$ is selected instead of the unknown optimal one of the actual day $t_{k}^{*}$ ? Using our notation, this difference is $C V_{k}\left(t_{k}^{*}\right)-C V_{k}\left(t_{1}^{*}\right)$, where $k>1$ indicates the day of interest. We show this quantity in the inset of each panel as the diff value. A value of diff $=0$ implies no change in optimal task-pair between the two considered days.

The mean of this value across all subjects is $2.2 \%$, statistically different from zero ( $\mathrm{p}$-value $=$ 0.038 ) indicating a significant loss. Note, however, that this is a worst case scenario, since the optimal task $t_{k}^{*}$ for day $k$ is unknown a priori. A more realistic scenario is to compare the diference in performance between selecting $t_{1}^{*}$ and a task-pair without any prior information. In this case, the benefit of task selection is significant ( $\mathrm{p}$-value $<0.003$ ). Selecting the task-pair without considering prior performance results in a mean loss value of $4.8 \%$, which doubles the loss of selecting $t_{1}^{*}$.

It is interesting to relate these results with the effect of subject learning previously described. Since learning might occur for all tasks, one would expect that the benefit of choosing $t_{1}^{*}$ on a following day would be attenuated with respect to random task-pair selection. Remarkably, we observe the opposite effect: for subjects with negative learning, e.g. B-S2 and B-S8, choosing $t_{1}^{*}$ also results in no improvement compared to random task-pair selection. In contrast, for those subjects with large learning values, e.g. subjects B-S4 and B-S9, the diff value attains higher values compared to random task-pair selection ( $9 \%$ and four times better, respectively).

Further, in some cases where learning is negative we observe a global decrease in performance, e.g. subjects C-S1 and C-S2 (day 3), which by having similar influence over all the task-pairs does not influence the optimality of $t_{1}^{*}$. Such a non task related decrease of the signal to noise ratio could be attributed to the presence of stronger non-stationarities in the subsequent day.

For subject C-S2, we compare the performances on the first day against each of the seven following days, resulting in six comparisons (right panel in third row and bottom panels of Figure 3). The learning of this subject is remarkable (circles lay above the diagonal in most of the panels). The optimal task-pair changes only in two days (days 4 and 6). Using $t_{1}^{*}$ as task-pair is generally convenient in this case.

Finally, we consider subject B-S5 for being the subject with worst performance and for which satisfactory BCI control would not be possible. In this case a clearly suboptimal task-pair became 
the optimal on the second day. For this kind of users, a task-pair selection step might be necessary on every single day, although not always sufficient for direct control.

From these results we conclude that the best task-pair selected from the first recording day of a given subject is generally a good task-pair on subsequent days. In general, task learning from the user side has a positive influence. However, special attention should be given to inexperienced subjects.

\section{Discussion}

We have analyzed the benefits of task selection for BCI on a large-scale basis (109 subjects) and have showed evidence that a significant proportion of users can potentially gain BCI control if the mental task is properly selected. Our results only consider three different tasks and better improvements can be expected for a larger number of tasks [13], or tasks that involve non-motor aspects [16]. Combining motor and non-motor strategies should allow some users unable to control motor imagery based BCIs to control binary devices. The on line validation of these results is part of current research. However, such non-motor imagery strategies might feel less natural for control and the subject learning process could still be hard [14].

Task selection is a key step towards BCI development, but is also a time consuming process. We have addressed some questions regarding the generalization of the optimal task-pair across days. Our initial results suggest that the loss of performance when optimal tasks are not individually selected in every session varies across subjects and is influenced by task learning from the subject side. Interestingly, we observed that the ability of the subject to learn tasks tends to minimize the expected loss when transferring optimal task-pairs from previous sessions.

Since the task selection method used in this work relies on the CV classification rate of an entire session and considers all combinations of task-pairs, it may be impractical in a real BCI setting, specially for a large number of tasks. Further, we have shown that the interplay between task learning from the user side and optimal task selection from the BCI side can be complex and consequently, task selection may require the use of exploration on every single day. The development of scalable adaptive methods that perform automatic task selection in a similar way as recently proposed [17] is the focus of current research. By focusing on the most promising tasks, these algorithms result in a faster task selection and a more efficient use of the BCI training session.

Our results show that one can transfer information from previous days to such algorithms but special care should be given to inexperienced subjects; previously suboptimal task-pairs can 
sometimes significantly improve its performance. Training strategies across days should be designed to estimate the subject learning curve as well as number of degrees of freedom each subject can control. The implementation of such protocols is the subject of current research. In on line experiments, the use of adaptive methods is required [39, 43, 35, 28, 1, 29]. Such adaptation strategies have been shown to be efficient when transferring classifiers across days or sessions for a fixed subject $[26,43]$. The use of these adaptive techniques have also shown that some subjects can learn to generate the adequate brain patterns after several sessions [42, 44, 45]. Adaptive

methods allow for an improved feedback which certainly influences and helps the subject learning process [5]. Even though the step from off line to on line is a complex process and many factors might influence the subject ability to control a final application, task selection makes the process simpler and consequently more satisfactory for the subject. Further research directed towards task validation, evaluation and selection is necessary.

\section{Acknowledgments}

The authors gratefully acknowledge the support of the Brain-Gain Smart Mix Programme of the Netherlands Ministry of Economic Affairs and the Netherlands Ministry of Education, Culture and Science.

\section{References}

[1] M. Arvaneh, C. Guan, K. K. Ang, and C. Quek. EEG Data Space Adaptation to Reduce Intersession Nonstationarity in Brain-Computer Interface. Neural Comput., 25(8):2146-2171, 2013.

[2] A. Barachant, S. Bonnet, M. Congedo, and C. Jutten. Common spatial pattern revisited by Riemannian geometry. In IEEE International Workshop on Multimedia Signal Processing (MMSP), pages 472-476, 2010.

[3] A. Barachant, S. Bonnet, M. Congedo, and C. Jutten. Multiclass Brain-Computer Interface classification by Riemannian geometry. IEEE Trans. Biomed. Eng., 59(4):920-928, 2012.

[4] F. Barbaresco. Innovative tools for radar signal processing based on cartans geometry of SPD matrices and information geometry. IEEE Radar Conference, 2008.

[5] A. Barbero Jimenez and M. Grosse-Wentrup. Biased feedback in brain-computer interfaces. J. NeuroEng. Rehabil., 7(34):1-4, 2010. 
[6] C. M. Bishop. Pattern Recognition and Machine Learning (Information Science and Statistics). Springer, Springer-Verlag New York, Inc., 1st edition, 2006.

[7] B. Blankertz, G. Dornhege, M. Krauledat, K. R. Müller, and G. Curio. The non-invasive Berlin BrainComputer Interface: Fast acquisition of effective performance in untrained subjects. NeuroImage, 37(2):539-550, 2007.

[8] B. Blankertz, S. Lemm, M. Treder, S. Haufe, and K.-R. Müller. Single-trial analysis and classification of ERP components - a tutorial. NeuroImage, 56(2):814-825, 2011.

[9] B. Blankertz, C. Sannelli, S. Halder, E. Hammer, A. Kübler, K. Müller, G. Curio, and T. Dickhaus. Neurophysiological predictor of SMR-based BCI performance. NeuroImage, 4(51):13031309, 2010.

[10] E. A. Curran and M. J. Stokes. Learning to control brain activity: A review of the production and control of EEG components for driving brain computer interface (BCI) systems. Brain Cog., 51(3):326 - 336, 2003.

[11] J. Demšar. Statistical comparisons of classifiers over multiple data sets. J. Mach. Learn. Res., 7(dec):1-30, 2006.

[12] M. P. do Carmo. Differential Geometry of Curves and Surfaces. Prentice-Hall, Englewood Cliffs, NJ, 1976.

[13] M. Dobrea and D. Dobrea. The selection of proper discriminative cognitive tasks; A necessary prerequisite in high-quality BCI applications. In 2nd International Symposium on Applied Sciences in Biomedical and Communication Technologies, ISABEL, pages 1-6, 2009.

[14] G. Dornhege, B. Blankertz, G. Curio, and K. R. Müller. Boosting bit rates in non-invasive EEG single-trial classifications by feature combination and multi-class paradigms. IEEE Trans. Biomed. Eng., 51(6):993-1002, 2004.

[15] P. Fletcher and S. Joshi. Principal geodesic analysis on symmetric spaces: Statistics of diffusion tensors. In Computer Vision and Mathematical Methods in Medical and Biomedical Image Analysis, volume 3117 of Lecture Notes in Computer Science, pages 87-98. Springer Berlin Heidelberg, 2004.

[16] E. V. Friedrich, R. Scherer, and C. Neuper. The effect of distinct mental strategies on classification performance for brain-computer interfaces. Int. J. Psychophysiol., 84(1):86 - 94, 2012.

[17] J. Fruitet, A. Carpentier, M. Clerc, and R. Munos. Bandit Algorithms boost Brain Computer Interfaces for motor-task selection of a brain-controlled button. In Advances in Neural Information Processing Systems 25, pages 458-466, 2012.

[18] A. L. Goldberger, L. A. N. Amaral, L. Glass, J. M. Hausdorff, P. C. Ivanov, R. G. Mark, J. E. Mietus, G. B. Moody, C.-K. Peng, and H. E. Stanley. PhysioBank, PhysioToolkit, and PhysioNet: 
Components of a new research resource for complex physiologic signals. Circulation, 101(23):e215e220, 2000.

[19] C. Guger, G. Edlinger, W. Harkam, I. Niedermayer, and G. Pfurtscheller. How many people are able to operate an EEG-based brain-computer interface (BCI)? IEEE Trans. Neural Syst. Rehabil. Eng., 11(2):145-147, 2003.

[20] L. F. Haas. Hans Berger (1873 - 1941), Richard Caton (1842 - 1926), and electroencephalography. J. Neurol. Neurosurg. Psychiatry, 74(1):9, 2003.

[21] E. Hammer, S. Halder, B. Blankertz, C. Sannelli, T. Dickhaus, S. Kleih, and K. Müller. Psychological predictors of SMR-BCI performance. Biol. Psychol., 89(1):80-86, 2012.

[22] S. T. Heath. A History of Greek Mathematics. Vol. 1. From Thales to Euclid. Dover classics of science and mathematics. Dover Publ., 1981.

[23] H. Karcher. Riemannian center of mass and mollifier smoothing. Commun. Pure Appl. Math., 30(5):509-541, 1977.

[24] Z. A. Keirn and J. I. Aunon. A new mode of communication between man and his surroundings. IEEE Trans. Biomed. Eng., 37(12):1209-1214, 1990.

[25] M. Krauledat. Analysis of Nonstationarities in EEG signals for improving Brain-Computer Interface performance. PhD thesis, Technische Universität Berlin, Fakultät IV - Elektrotechnik und Informatik, 2008.

[26] M. Krauledat, M. Tangermann, B. Blankertz, and K.-R. Müller. Towards zero training for braincomputer interfacing. PLoS ONE, 3(8):e2967, 082008.

[27] A. Kübler, N. Neumann, B. Whilhelm, T. Hinterberger, and N. Birbaumer. Predictability of braincomputer communication. J. Psychophysiol., 18(2-3):121-129, 2004.

[28] A. Llera, V. Gómez, and H. J. Kappen. Adaptive classification on brain computer interfaces using reinforcement signals. Neural Comput., 24(11):2900-2923, November 2012.

[29] A. Llera, V. Gómez, and H. J. Kappen. Adaptive multi class classification for brain computer interfaces. Neural Comput., 26(6):1108-1127, June 2014.

[30] M. Moakher. A differential geometric approach to the geometric mean of symmetric positive-definite matrices. SIAM J. Matrix Anal. Appl., 26(3):735-747, 2005.

[31] M. Moakher and M. Zéraï. The Riemannian geometry of the space of positive-definite matrices and its application to the regularization of positive-definite matrix-valued data. J. Math. Imaging Vis., 40(2):171-187, 2011.

[32] B. Obermaier, C. Neuper, C. Guger, and G. Pfurtscheller. Information transfer rate in a five-classes brain-computer interface. IEEE Trans. Neural Syst. Rehabil. Eng., 9(3):283-288, 2001. 
[33] G. Pfurtscheller, C. Neuper, D. Flotzinger, and M. Pregenzer. EEG-based discrimination between imagination of right and left hand movement. Electroencephalogr. Clin. Neurophysiol., 103(6):642$651,1997$.

[34] H. Ramoser, J. Müller-Gerking, and G. Pfurtscheller. Optimal spatial filtering of single trial EEG during imagined hand movement. IEEE Tran. Rehabil. Eng., 8:441-446, 1998.

[35] B. Reuderink, J. Farquhar, M. Poel, and A. Nijholt. A subject-independent brain-computer interface based on smoothed, second-order baselining. In Proceedings of the 33st Annual International Conference of the IEEE Engineering in Medicine and Biology Society (EMBC 2011), pages 4600-4604, 2011.

[36] G. Schalk, D. J. Mcfarl, T. Hinterberger, N. Birbaumer, and J. R. Wolpaw. BCI2000: A generalpurpose brain-computer interface (BCI) system. IEEE Trans. Biomed. Eng., 51(6):1034-1043, 2004.

[37] P. Shenoy, M. Krauledat, B. Blankertz, R. P. Rao, and K.-R. Müller. Towards adaptive classification for BCI. J. Neural Eng., 3(1):R13, March 2006.

[38] M. Tangermann, K. R. Müller, A. Aertsen, N. Birbaumer, C. Braun, C. Brunner, R. Leeb, C. Mehring, K. Miller, G. Müller-Putz, G. Nolte, G. Pfurtscheller, H. Preissl, G. Schalk, A. Schlögl, C. Vidaurre, S. Waldert, and B. Blankertz. Review of the BCI competition IV. Front. Neurosci., 6(55):1-31, 2012.

[39] R. Tomioka, J. Hill, B. Blankertz, and K. Aihara. Adapting spatial filter methods for nonstationary BCIs. In Proceedings of 2006 Workshop on Information-Based Induction Sciences (IBIS 2006), pages 65-70. Max-Planck-Gesellschaft, 112006.

[40] M. van Gerven and O. Jensen. Attention modulations of posterior alpha as a control signal for twodimensional brain-computer interfaces. J. Neurosci. Methods, 179(1):78 - 84, 2009.

[41] J. J. Vidal. Toward Direct Brain-Computer Communication. Annu. Rev. Biophys. Bioeng., 2:157-180, 1973.

[42] C. Vidaurre and B. Blankertz. Towards a cure for BCI illiteracy. Brain Topogr., 23(2):194-198, 2010.

[43] C. Vidaurre, M. Kawanabe, P. von Bünau, B. Blankertz, and K. Müller. Toward an unsupervised adaptation of LDA for Brain-Computer Interfaces. IEEE Trans. Biomed. Eng., 58(3):587 - 597, 2011.

[44] C. Vidaurre, C. Sannelli, K. Müller, and B. Blankertz. Co-adaptive calibration to improve BCI efficiency. J. Neural Eng., 8(2):025009, 2011.

[45] C. Vidaurre, C. Sannelli, K.-R. Müller, and B. Blankertz. Machine-learning-based coadaptive calibration for brain-computer interfaces. Neural comput., 23(3):791-816, 2011.

[46] J. R. Wolpaw, N. Birbaumer, D. J. McFarland, G. Pfurtscheller, and T. M. Vaughan. Brain-computer interfaces for communication and control. Clin. Neurophysiol., 113(6):767-791, June 2002. 